\title{
Clinical and Imaging Features of Anterior Choroidal Artery Infarction
}

Guang-Sheng Wang ${ }^{1}$, Ting Hu${ }^{1}$, Jinjian Yang ${ }^{1}$, Yuanyuan Tian ${ }^{1}$, Li Huang ${ }^{1}$, Yuanwei Wang $^{1}$, Hanpei $\mathrm{Gu}^{1}$, and Ying Wang ${ }^{1}$

${ }^{1}$ Affiliated Shuyang People's Hospital of Xuzhou Medical University

September 24, 2021

\begin{abstract}
Infarct Size, Imaging Features and Stroke Types with Different Clinical Types of Anterior Choroidal Artery Blood Supply Area Infarction
\end{abstract}

\section{Hosted file}

AChAClinicalResearch_WangGuangSheng.doc available at https://authorea.com/users/435006/ articles/538323-clinical-and-imaging-features-of-anterior-choroidal-artery-infarction

\section{Hosted file}

Figure1.docx available at https://authorea.com/users/435006/articles/538323-clinical-andimaging-features-of-anterior-choroidal-artery-infarction

\section{Hosted file}

Figure2.docx available at https://authorea.com/users/435006/articles/538323-clinical-andimaging-features-of-anterior-choroidal-artery-infarction

\section{Hosted file}

Figure3.docx available at https://authorea.com/users/435006/articles/538323-clinical-andimaging-features-of-anterior-choroidal-artery-infarction 